\title{
Perinatal Tuberculosis: A Diagnostic Challenge
}

\author{
Edna Lúcia S. de Souza, Lícia Maria O. Moreira \\ and Márcio Fernando Silva
}

\author{
Department of Pediatrics, Federal University of Bahia; \\ Salvador, BA, Brazil
}

\begin{abstract}
Despite the high prevalence of tuberculosis in adults and children, the congenital and perinatal forms of tuberculosis are rare. In Brazil, there has been only one published case of congenital tuberculosis and two cases of the perinatal form of this disease. We report a case of perinatal tuberculosis presenting with pneumonia. Alcohol-acid-resistant bacilli were found in the gastric lavage. Diagnosis of this disease presentation requires a high index of suspicion.

Key Words: Congenital tuberculosis, perinatal tuberculosis, tuberculosis, pneumonia.
\end{abstract}

Tuberculosis is a serious public health issue. According to World Health Organization (WHO) statistics, around 8.8 million new cases of the disease occurred worldwide in 2003 [1]. Several factors, such as the HIV pandemic, widespread population impoverishment, appearance of multi-resistant strains of this disease, migratory flows, and inefficient health programs, have contributed towards aggravating the problem. Despite the high prevalence of this disease in adults and children, the congenital and perinatal forms of tuberculosis are rare. Around 300 cases of the congenital form have been reported, and only sparse reports exist on the perinatal form. A Medline search identified only three cases of perinatal tuberculosis published between 2000 and 2004. In 2005, up to the present moment, reports on two cases of congenital tuberculosis and one case of perinatal tuberculosis have been published. In Brazil, there has been only one published case of congenital tuberculosis [2] and two cases of the perinatal form of this disease [3]. Considering the impact of infectious diseases on perinatal morbidity and mortality, and the high prevalence of tuberculosis in women, this possibility should be considered when diagnosing women of reproductive age. We describe a case of perinatal tuberculosis in a child with symptoms beginning on the 15 th day of life.

\section{Case Report}

A 48-day old child was transferred to the very young infants unit of the pediatric department of the University Teaching Hospital because of fever and extensive pneumonia in the right hemithorax, which failed to improve despite the use of various antibiotic regimens. The child had been hospitalized in another institution since his 15th day of life because of fever and dyspnea that developed into pneumonia and led to poor weight gain. The child was born at the $8^{\text {th }}$

Received on 28 September 2005; revised 29 January 2006.

Address for correspondence: Dr. Edna Lúcia Santos de Souza. Av. Paulo VI, 2200, apt. 104, Itaigara, Salvador, Bahia, Brazil. Zip code:41.810.001. Phone: +55 71-3358 3326. Fax: +55 71-3362 4000. E-mail: ednalu@ufba.bror ednalss@hotmail.com

The Brazilian Journal of Infectious Diseases 2006;10(3):228-229. (C) 2006 by The Brazilian Journal of Infectious Diseases and Contexto Publishing. All rights reserved. month of pregnancy, with no health complications, weighing 2,005 grams (low birth-weight), and was released from the hospital at the same time as his mother. The child received BCG vaccination at birth and was exclusively breastfed for only three days.

Physical examination detected tachypnea, pallor and malnutrition, with a respiratory rate of $78 \mathrm{bpm}$ and a heart rate of $140 \mathrm{bpm}$. Respiratory auscultation detected a reduction in vesicular murmur in the right hemithorax and the presence of crepitus in the right. The liver was palpable $5 \mathrm{~cm}$ from the right costal margin and xiphoid process and the spleen $5 \mathrm{~cm}$ from the left costal margin. Results of full blood counts were as follows: hemoglobin $8.5 \mathrm{~g} / \mathrm{dL}$, hematocrit $25.9 \%$, WBC count $17,000 / \mathrm{mm}^{3}, 23 \%$ banded neutrophils, 30\% segmented neutrophils, $11 \%$ monocytes, 33\% lymphocytes, and 5\% eosinophils. Blood culture, urinalysis, ToRCHS and HIV testing were negative. Tuberculin test was non-reactive. Chest $\mathrm{x}$-ray showed extensive homogenous condensation in the right hemithorax (Figure 1). Transfontanel and abdominal ultrasonography were carried out, and the results were normal. Computerized tomography of the thorax revealed areas of condensation in the right upper lobe and both inferior lobes, with an air-filled bronchogram and areas suggestive of necrosis (Figure 2). Alcohol-acid-resistant bacilli were detected in the gastric lavage on the $6^{\text {th }}$ day of hospitalization, and treatment with isoniazid, rifampicin and pyrazinamide was initiated. Cerebral-spinal fluid culture was normal. Gastric lavage culture was positive for Mycobacterium tuberculosis 56 days after sample collection. The child became afebrile six days after initiating tuberculosis treatment, began to gain weight and progressively improved. However, he still had insufficient suckling at breastfeeding, which prolonged his stay in the hospital. The baby was released after four months of hospitalization in good clinical condition and is currently being followed up as an outpatient in the Pediatric Pneumology Department. He continues to gain weight but has poor weight for height development. The child's mother had failed to attend regular prenatal care and reported fever that began during the seventh month of pregnancy. She was readmitted to the hospital five days after delivery due to a foul-smelling vaginal discharge, fever and cough that progressed to respiratory failure. She was submitted to various antibiotic regimens, showed a slight clinical improvement but continued febrile. 
Figure 1. Chest x-ray- Extensive condensation in the right hemithorax

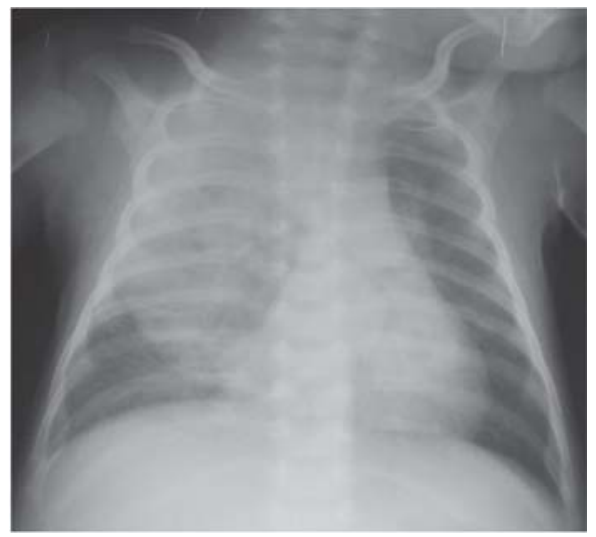

She underwent chest $\mathrm{x}$-ray, bronchoalveolar lavage, pulmonary scintillography, anti-HIV testing and bronchoscopy, and no abnormality was found. A transbronchial biopsy was carried out and the results revealed irregular septal fibrosis, irregular focal emphysema and isolated, non-caseating pulmonary granuloma. Tuberculosis therapy was initiated and the patient responded well to treatment.

\section{Discussion}

The criteria currently used for the diagnosis of congenital tuberculosis are: tuberculosis confirmed in the child, plus at least one of the following findings: 1) lesions during the first week of life; 2) primary hepatic complex or caseous hepatic granuloma; 3) tuberculosis infection in the placenta or in the genital tract; 4) exclusion of the possibility of post-natal transmission following investigation of all contacts [4].

According to the literature, the symptoms generally begin in the second or third week of life, and most commonly include fever, low weight gain, irritability, respiratory distress, poor feeding and hepatosplenomegaly [5-8].

Evidence points towards a congenital form of tuberculosis in our patient: the mother was ill during pregnancy and became worse in the post-partum period; she responded satisfactorily to specific treatment for tuberculosis; the child had early symptoms beginning on the $15^{\text {th }}$ day of life, and contact with his mother occurred only during the first five days of life.

The two routes of acquisition of congenital tuberculosis are: 1) hematogenic, through the umbilical vein, with a primary lesion in the liver or lung; and 2) breathing or swallowing infected material, with the primary lesion in the lung or gastrointestinal tract [8,9]. Unfortunately, in this case no evaluation was carried out in the genital tract of the mother; therefore, we are unable to affirm that transmission occurred congenitally and perinatal transmission of the disease must be assumed.

This case emphasizes the need to consider congenital or perinatal tuberculosis when diagnosing infants with pneumonia that is non-responsive to adequate antibiotic
Figure 2. Computerized tomography of the thorax - areas of condensation in the right upper lobe and both inferior lobes, with air-filled bronchogram and areas suggestive of necrosis

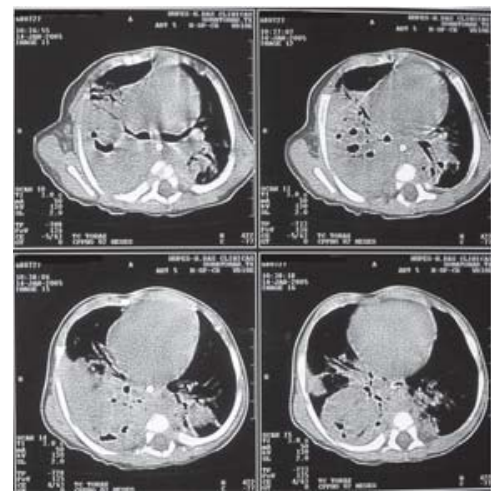

regimens, with fever of unidentified origin and/or hepatosplenomegaly and/or poor feeding and irritability and/ or failure to gain weight. Early diagnosis of this condition is essential to improve prognosis since the mortality rate is around $100 \%$ in untreated children $[6,8]$ and $22 \%$ amongst those who receive treatment [4]. Fortunately, this patient had a favorable outcome despite the late diagnosis. The disease may be under-diagnosed due to its wide range of clinical variations that contribute to the paucity of reported cases. Diagnostic investigation of the congenital or perinatal forms of this disease should be considered, particularly in countries such as Brazil, where there is a high prevalence of tuberculosis.

\section{Acknoeledgements}

We thank Dr. Candida Athaide e Oliveira for taking care of the child.

\section{References}

1. World Health Organization. Global Tuberculosis Controlsurveillance, planning, enhancing. Available from: http// www.who.int/tb/publications/global_report/2005. Accessed on September 25, 2005.

2. Ferlin M.L.S., Bissani C., Jorge S.M., et al. Tuberculose congênita. J Pediatr. 1998;74:239-42.

3. Souza W.A.S., Feldens C.E., Lueska S., Miura E. Tuberculose perinatal: relato de dois casos. Revista HCPA 1991;11:170-2.

4. Cantwell M.F., Shehab Z.M., Costello A.M., et al. Congenital tuberculosis. N Engl J Med 1994;330:1051-4.

5. Chotpitayasunondh T., Sangtawesin V. Congenital tuberculosis. Med Assoc Thai 2003;86:S689-S95.

6. Hageman J., Shulman S., Schreiber M., et al. Congenital tuberculosis: Critical reappraisal of clinical findings and diagnostic procedures. Pediatrics 1980;66:980-4.

7. Jacobs R.F., Abernathy R.S. Management of tuberculosis in pregnancy and the newborn. Clin Perinatol 1988;15:305-19.

8. Starke J.R. Tuberculosis: an old disease but a new threat to the mother, fetus, and neonate. Clin Perinatol 1997;24:107-25.

9. Vallejo J.G., Starke J.R. Tuberculosis and pregnancy. Clin Chest Med 1992;13:693-707. 